\title{
Association study of polymorphisms in interferon- $\gamma$ receptor genes with the risk of pulmonary tuberculosis
}

\author{
JOONG-GON SHIN $^{1 *}$, BYUNG LAE PARK ${ }^{2 *}$, LYOUNG HYO KIM ${ }^{1,2}$, SUHG NAMGOONG $^{1,2}$, JI ON KIM ${ }^{1,2}$, \\ HUN SOO CHANG ${ }^{3,4}$, JONG SOOK PARK ${ }^{4}$, AN SOO JANG ${ }^{4}$, SUNG WOO PARK $^{4}$, DO JIN KIM ${ }^{4}$, KI UP KIM ${ }^{5}$, \\ YANG GEE KIM ${ }^{5}$, SOO-TAEK UH ${ }^{5}$, KI HYUN SEO ${ }^{6}$, YOUNG HOON KIM ${ }^{6}$, \\ INSONG $\mathrm{KOH}^{7}, \mathrm{CHOON}^{\text {SIK PARK }}{ }^{4}$ and HYOUNG DOO SHIN ${ }^{1,2}$
}

${ }^{1}$ Department of Life Science, Sogang University, Seoul 121-742; ${ }^{2}$ Department of Genetic Epidemiology, SNP Genetics, Inc., Seoul 121-742; ${ }^{3}$ Soonchunhyang Medical Science Research Institute, Soonchunhyang University Bucheon Hospital;

${ }^{4}$ Genome Research Center for Allergy and Respiratory Diseases, Division of Allergy and Respiratory Medicine, Soonchunhyang University Bucheon Hospital, Bucheon, Gyeonggi-do 420-020; ${ }^{5}$ Division of Allergy and Respiratory Medicine, Soonchunhyang University Seoul Hospital, Seoul 140-743; ${ }^{6}$ Division of Allergy and Respiratory Medicine, Soonchunhyang University, Cheonan Hospital, Cheonan, Hoseo 30240; ${ }^{7}$ Department of Physiology,

Hanyang University College of Medicine, Seoul 133-791, Republic of Korea

Received May 29, 2014; Accepted February 23, 2015

DOI: $10.3892 / \mathrm{mmr} .2015 .3544$

\begin{abstract}
Tuberculosis (TB) is an infectious disease caused by mycobacterium, which most commonly affects the lungs. The adaptive immune response in Mycobacterium tuberculosis is predominantly mediated by the interferon- $\gamma($ IFN- $\gamma)$ signaling pathway, which is regulated by IFN $-\gamma$ receptors (IFNGR). IFN- $\gamma$ activates the transcription of a number of genes that are important in immune responses, thus the appropriate function of IFNGR appears to be important in host defense against mycobacteria. In the present study, 22 genetic variants in IFNGRI and IFNGR2 were genotyped in 673 patients and 592 normal controls to investigate the association between IFNGR1 and IFNGR2 polymorphisms and the risk of TB. Statistical analyses revealed that four genetic variants in IFNGR1, rs9376269, rs9376268, rs9376267 and rs56251346 were marginally associated with the risk of TB $(\mathrm{P}=0.02-0.04)$, while other single nucleotide polymorphisms in IFNGRI
\end{abstract}

Correspondence to: Professor Hyoung Doo Shin, Department of Life Science, Sogang University, 1 Shinsu-dong, Mapo-gu, Seoul 121-742, Republic of Korea

E-mail: hdshin@sogang.ac.kr

Professor Choon Sik Park, Genome Research Center for Allergy and Respiratory Diseases, Division of Allergy and Respiratory Medicine, Soonchunhyang University Bucheon Hospital, 1174 Jung-Dong, Wonmi-Ku, Bucheon, Gyeonggi-Do 420-020, Republic of Korea

E-mail: mdcspark@hanmail.net

*Contributed equally

Key words: single nucleotide polymorphism, interferon- $\gamma$ receptor 1 , interferon- $\gamma$ receptor 2 , tuberculosis and IFNGR2 did not exhibit any associations. However, the significance of the four genetic variants rs9376269, rs9376268, rs9376267 and rs56251346 was eliminated following a multiple testing correction of the data $(\mathrm{P}>0.05)$. The present results revealed that certain genetic variants in IFNGR genes may be associated with TB development, which may be useful preliminary data for future investigation.

\section{Introduction}

Tuberculosis (TB) is an infectious disease commonly caused by mycobacteria (1). TB is considered to be an acute global health problem with $\sim 9$ million novel TB cases and 1.4 million fatalities each year (2). TB commonly originates in the lungs, but is able to spread to other parts of the body, leading to extra-pulmonary diseases (3). Among the patients infected with $\mathrm{TB}, \sim 10 \%$ progress to active TB during their lifespan and the remaining individuals remain asymptomatic (4). The immune responses of TB patients are mainly regulated by $\mathrm{T}$ helper 1 cells, which secrete interferon- $\gamma($ IFN- $\gamma$ ) (5). IFN- $\gamma$ mediated immune responses activate macrophages, which induce the secretion of other cytokines, including interleukin (IL)-1, IL-12 and tumor necrosis factor (TNF)- $\alpha$ (6). Previously, genome-wide association studies have revealed that genetic variation in genes involved in immune responses, including IL-1, IL-12 and TNF- $\alpha$, is associated with the risk of TB (7-9).

The IFN- $\gamma$-induced signaling pathway is activated by interacting with its receptor composed of two subunits, IFN- $\gamma$ receptor (IFNGR) 1 and 2, which encode the ligand-biding chain ( $\alpha$-chain) and the non-ligand binding chain, respectively. IFNGR is involved in a positive feedback loop of IFN- $\gamma$ expression (10). Genetic variation in cytokine-associated genes, including IFNGRI and IFNGR2, have previously been found to be important in other viral/host-mediated immune responses in TB (11-16). Among the genetic variants in IFNGRl, the 
A

Map of IFNGR1 (interferon gamma receptor 1) on chromosome 6q23.3 (21.94 kb) NM_000416.2

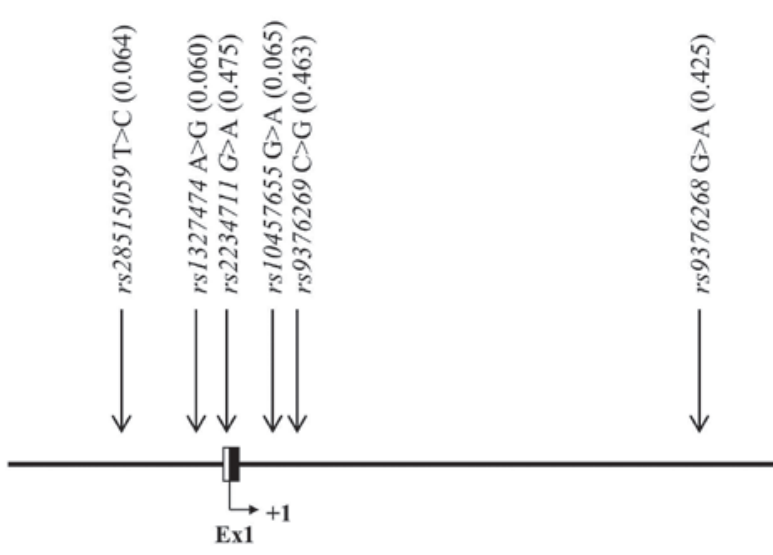

$\mathbf{B}$

Haplotype of IFNGRI

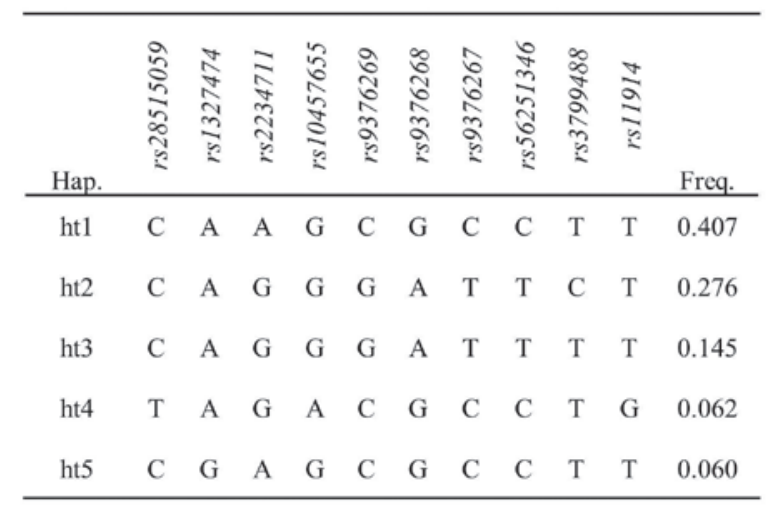

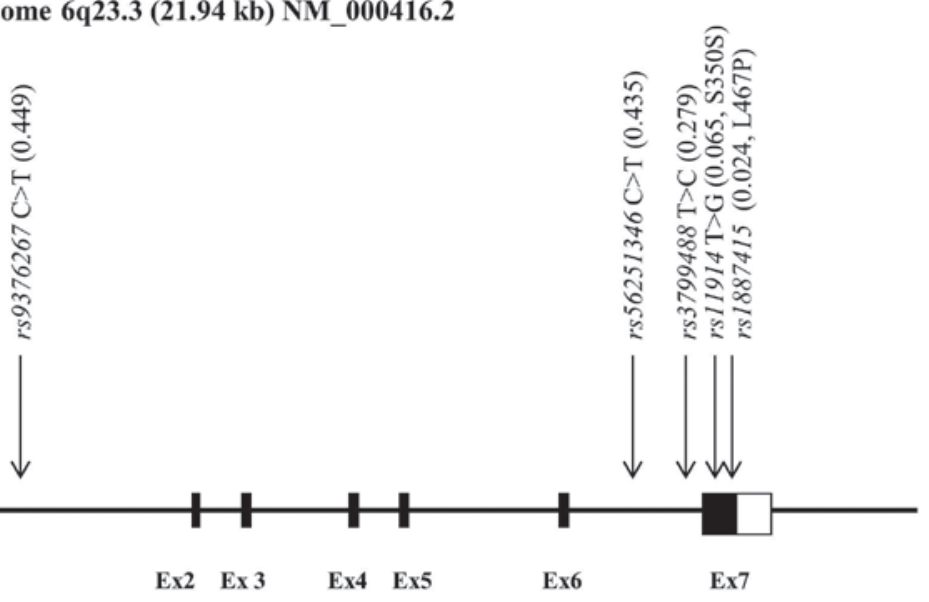

C

LDs among IFNGR1 polymorphisms

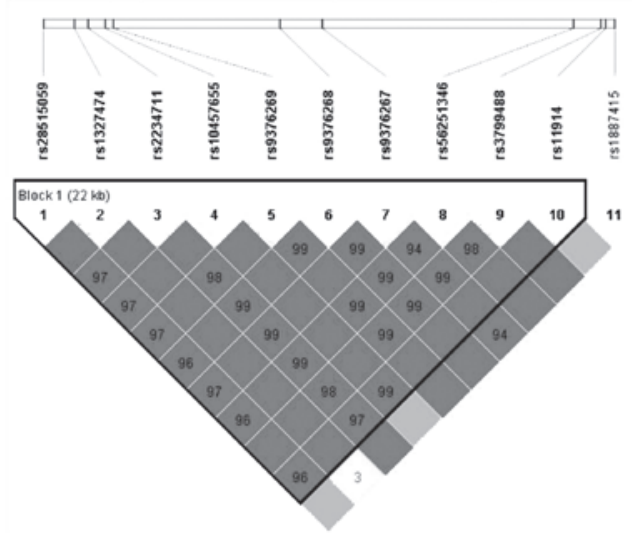

Figure 1. Schematic physical map of IFNGR1. (A) Polymorphisms of IFNGR1. Black blocks indicate coding exons; white blocks indicate 5'- and 3'-untranslated regions. First base of translation site is denoted as nucleotide +1 . (B) Haplotypes of IFNGR1 in a Korean population. (C) LDs among IFNGR1 polymorphisms. IFNGR, interferon- $\gamma$ receptors; LD, linkage disequilibrium; ht, haplotype.

single nucleotide polymorphism (SNP) $r$ s2234711 has been revealed to be a major marker of disease protection. In a recent Chinese study, patients with rs2234711 had a significantly lower prevalence of TB [odds ratio $(\mathrm{OR})=0.82, \mathrm{P}<0.001$ ] (17). However, to date, an association between the risk for TB and genetic variation in the IFNGRI and IFNGR2 genes had not been demonstrated in a Korean population. In the present study, the association of polymorphisms in the IFNGRI and IFNGR2 genes with the risk of TB in the Korean population was investigated.

\section{Patients and methods}

Patients. A total of 673 patients with clinical manifestation of pulmonary TB (mean age, 45.81 years; range, 16-92 years, 388 males and 285 females) were recruited from Soonchunhyang University Bucheon Hospital (Bucheon, Republic of Korea). Polymerase chain reaction was used to assess all sputum acid-fast bacillus culture-positive samples to distinguish Mycobacterium tuberculosis (MTB) from non-tuberculous mycobacteria (NTM). The diagnosis of pulmonary TB was confirmed by the isolation of MTB from the sputum or bronchoalveolar lavage fluid. Patients with an
NTM infection were excluded from the present study. Patients with TB who had a family history of the disease were also excluded from the study to eliminate the additional risk factors of added exposure to TB. A total of 592 healthy controls (mean age, 50.22 years; range, 9-87 years, 277 males and 315 females) were simultaneously recruited from a randomly sampled population who had attended the clinic for routine health checkups in the same regional area. Only patients above the age of 40 years were included in the normal control group to exclude the possibility of TB infection among young individuals who may subsequently develop the condition. Individuals with other medical diseases/conditions, including human immunodeficiency virus, hepatitis, diabetes, alcoholism, autoimmune diseases and cancer were also excluded from the present study.

The ethnicity of all patients and controls was Korean. Written informed consent was obtained from all patients prior to the start of the experiment. The experimental protocol was approved by the Institutional Review Board of Soonchunhyang University Bucheon Hospital (IRB no. schbc-biobank-2012-001).

SNP genotyping. Candidate SNPs of the IFNGRI and IFNGR2 genes were selected from Japanese and 

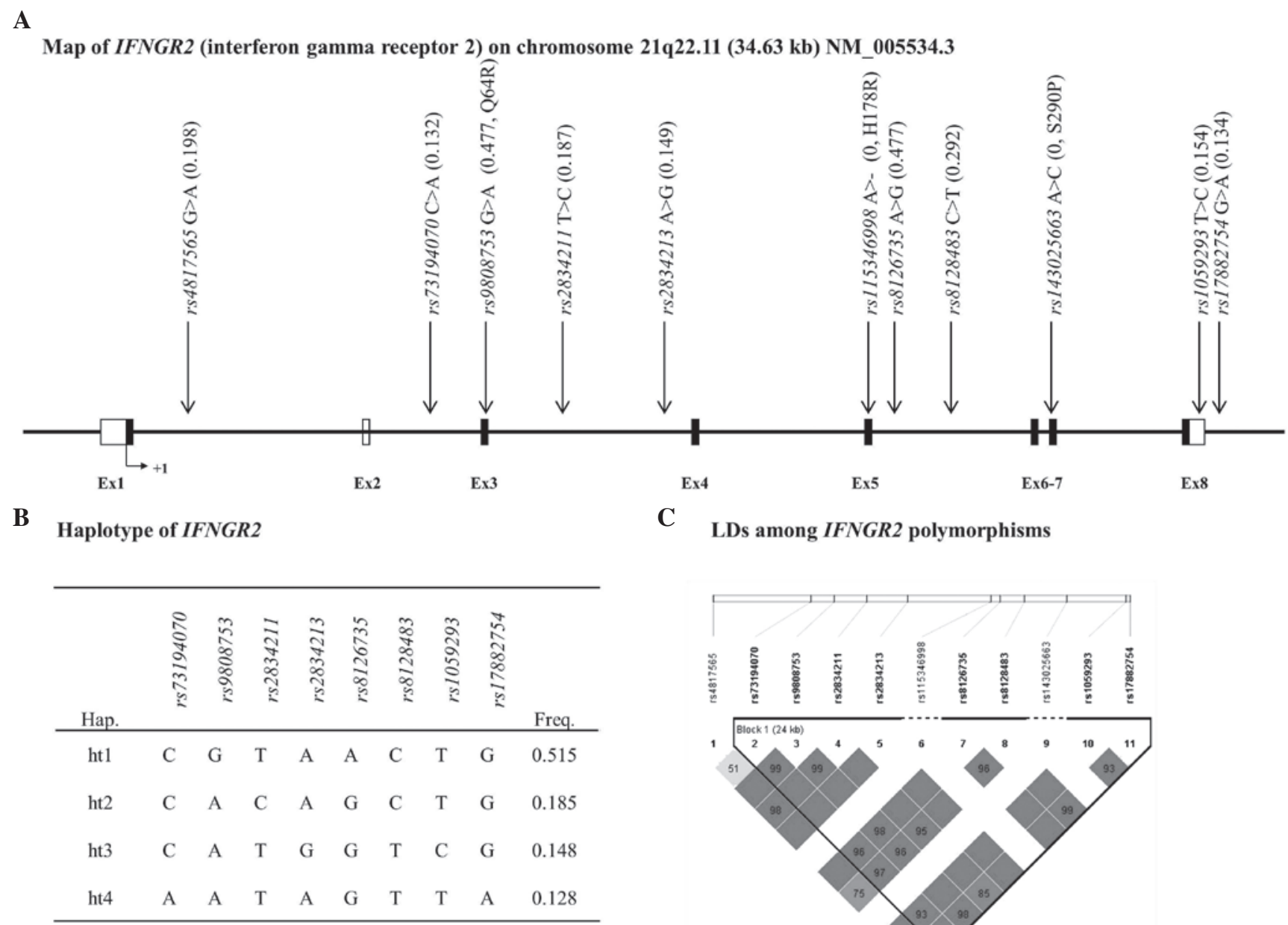

Figure 2. Schematic physical map of IFNGR2. (A) Polymorphisms of IFNGR2. Black blocks indicate coding exons; white blocks indicate 5'- and 3'-untranslated regions. First base of translation site is denoted as nucleotide +1 . (B) Haplotypes of IFNGR2 in a Korean population. (C) LDs among IFNGR2 polymorphisms. IFNGR, interferon- $\gamma$ receptors; LD, linkage disequilibrium; Ex, exon; ht, haplotype.

Han Chinese data from the 1,000 Genomes database (http://browser.1000genomes.org/index.html) based on the allele frequency and linkage disequilibrium (LD) status in the Asian population. Additional SNPs which had been previously investigated were also selected (14). A total of 11 SNPs of the IFNGRI gene and 11 SNPs of the IFNGR2 gene were selected based on the following criteria: Minor allele frequency (MAF; $>5 \%)$ and $\mathrm{LD}\left(r^{2}>0.98\right)$. A total of 22 polymorphisms were genotyped in $673 \mathrm{~TB}$ patients and 592 normal controls using a TaqMan assay on the ABI prism $7900 \mathrm{HT}$ sequence detection system (Applied Biosystems, Foster City, CA, USA) (18). Quality control of the genotyping was performed in $10 \%$ of the samples by duplicate checking (rate of concordance in duplicates, $>99.5 \%$ ). Selected SNPs and probe information on the polymorphisms is shown in Table I.

Statistical analysis. The level of LD was obtained using Haploview version 4.2 software (Broad Institute, Cambridge, MA, USA; http://www.broadinstitute.org/mpg/haploview), with examination of Lewontin's D' (ID'I) and the LD coefficient $r^{2}$ between all pairs of bi-allelic loci (19). Haplotypes were estimated using PHASE version 2.1 software (Stephen Laboratory, University of Chicago, Chicago, IL, USA) (20).
A comparison of genotype distributions between TB patients and healthy controls was performed using a logistic regression model adjusted for age (continuous value) and gender (male $=0$, female $=1$ ) as co-variates using SAS software, version 9.3 (SAS Inc., Cary, NC, USA). The effective number of independent marker loci was calculated for multiple testing corrections using SNPSpD (http://genepli.qimr.edu. $\mathrm{au} /$ general/daleN/SNPSpD/), a program based on the spectral decomposition of matrices of pair-wise LD between SNPs (21). The total sum of independent marker loci in the gene was calculated as 7.7553 for IFNGRI and 9.3328 for IFNGR2, and this value was applied to correct for multiple testing.

\section{Results}

Genotyping and haplotype analysis of IFNGRI and IFNGR2. In the present study, a total of 22 polymorphisms (11 in IFNGRI and 11 in IFNGR2) were selected, based on their MAF, location and LD status, and genotyped in $673 \mathrm{~TB}$ cases and 592 healthy controls. Detailed information regarding polymorphisms, including allele, amino acid change, position, MAF, heterozygosity and P-values for the Hardy-Weinberg equilibrium are shown in Table II. LDs among SNPs were 
Table I. Probe information for IFNGR1 and IFNGR2.

\begin{tabular}{|c|c|c|}
\hline Gene & Loci & Assay on demand ID or probe sequence \\
\hline \multirow[t]{14}{*}{ IFNGRI } & $r s 28515059$ & C__63095558_10 \\
\hline & rs 1327474 & C___2523634_10 \\
\hline & $r s 2234711$ & C__11693991_10 \\
\hline & rs 10457655 & C__30506149_10 \\
\hline & rs9376269 & C__30272193_20 \\
\hline & rs9376268 & C__30470198_10 \\
\hline & rs9376267 & C__30182293_10 \\
\hline & $r s 56251346$ & TGTTTACAAAGTGGGCACATC ${ }^{\mathrm{a}}$ \\
\hline & & ATTGGAAACATTTCCCCATC ${ }^{\mathrm{b}}$ \\
\hline & & CATTACTTGC \\
\hline & & CATTATTTGC $^{\mathrm{d}}$ \\
\hline & $r s 3799488$ & C__25647358_10 \\
\hline & $r s 11914$ & C___7578627_10 \\
\hline & $r s 1887415$ & C__11693851_30 \\
\hline \multirow[t]{32}{*}{ IFNGR2 } & $r s 4817565$ & GACATTGCCACAACATCCAG ${ }^{\mathrm{a}}$ \\
\hline & & GAGCCTGGCCTCACTTTTTA ${ }^{\mathrm{b}}$ \\
\hline & & ACCTGTCCAT $^{\mathrm{c}}$ \\
\hline & & ACCTATCCAT $^{\mathrm{d}}$ \\
\hline & rs73194070 & ACTGTGAGGGAGCATTGACC ${ }^{a}$ \\
\hline & & CCGAAGGCAGACAGGTAAAG \\
\hline & & $\mathrm{ACCACCCCCC}^{\mathrm{c}}$ \\
\hline & & ACCACACCCC ${ }^{\mathrm{d}}$ \\
\hline & rs9808753 & C___2443413_1_ \\
\hline & $r s 2834211$ & C__16072862_10 \\
\hline & $r s 2834213$ & C___2443417_10 \\
\hline & rs 115346998 & AGAAGGCTCCCTCATCATCA $^{\mathrm{a}}$ \\
\hline & & TCTTGCCTGTTGGATTCCTC \\
\hline & & TGTCCATTAC \\
\hline & & TGTCCGTTAC $^{\mathrm{d}}$ \\
\hline & $r s 8126735$ & TGAAGCATCTCCAGTGCCTA ${ }^{\mathrm{a}}$ \\
\hline & & GAGCCAAACACAAAGGAAGC $^{\mathrm{b}}$ \\
\hline & & TTATAATGGT $^{\mathrm{c}}$ \\
\hline & & TTATGATGGT $^{\mathrm{d}}$ \\
\hline & $r s 8128483$ & GAAGAGGCACATGGAGGAAA $^{\mathrm{a}}$ \\
\hline & & CCTGGCAGACAACAGTTCAC \\
\hline & & TCATCGCTCC ${ }^{\mathrm{c}}$ \\
\hline & & TCATTGCTCC $^{\mathrm{d}}$ \\
\hline & $r s 143025663$ & GTTTCACACTCCACCAAGCA $^{\mathrm{a}}$ \\
\hline & & GCTGCAGTGAGCAGAGATTG ${ }^{\mathrm{b}}$ \\
\hline & & TTACAGATAG $^{\mathrm{c}}$ \\
\hline & & TTACCGATAG $^{\mathrm{d}}$ \\
\hline & rs 1059293 & C___2443435_10 \\
\hline & rs 17882754 & TCATGGGAACTCAGCAAACA ${ }^{\mathrm{a}}$ \\
\hline & & CTCAAGTGATCCACCCACCT ${ }^{\mathrm{b}}$ \\
\hline & & CAGGGCCTAG $^{\mathrm{c}}$ \\
\hline & & CAGGACCTAG $^{\mathrm{d}}$ \\
\hline
\end{tabular}




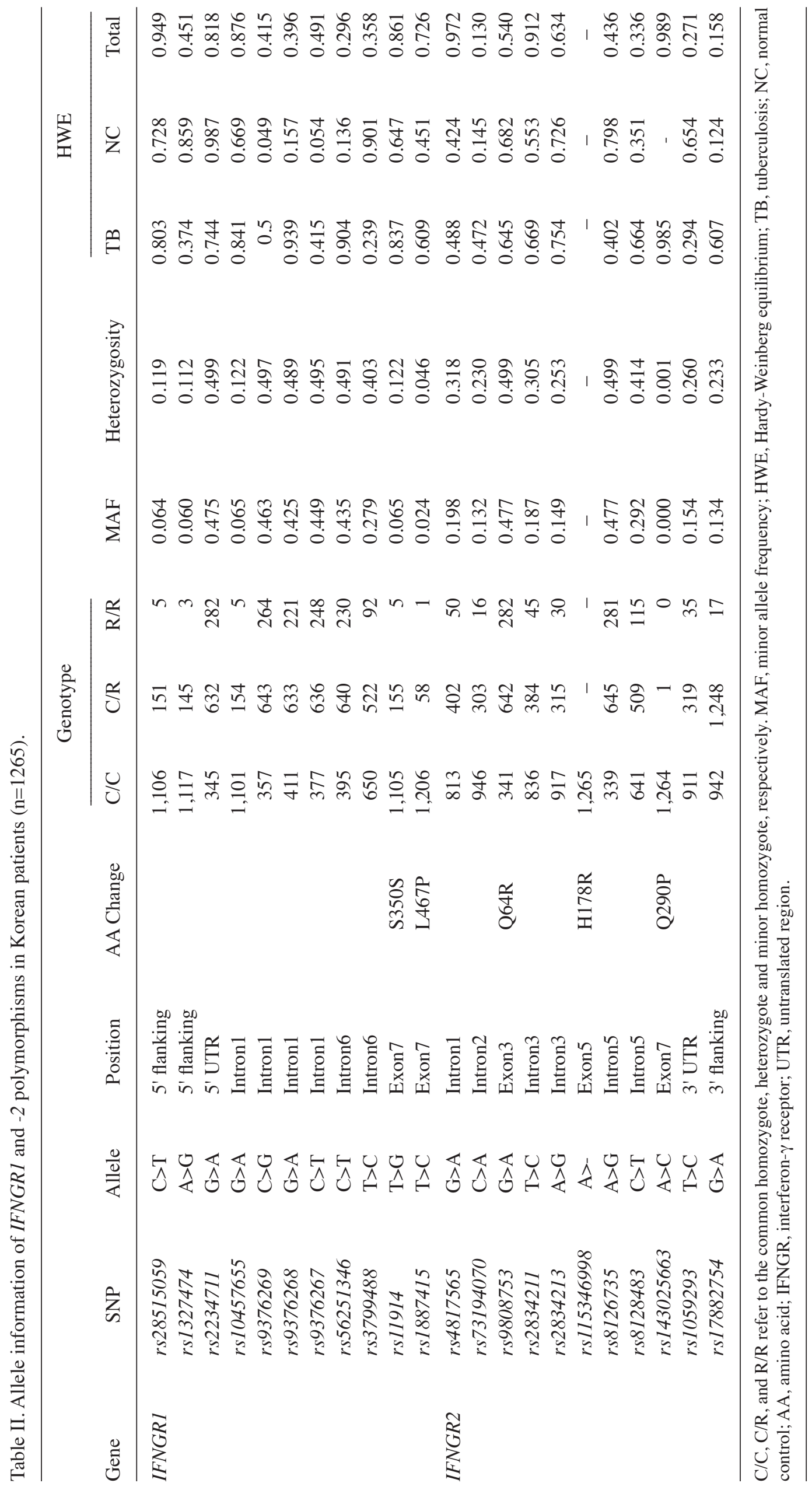




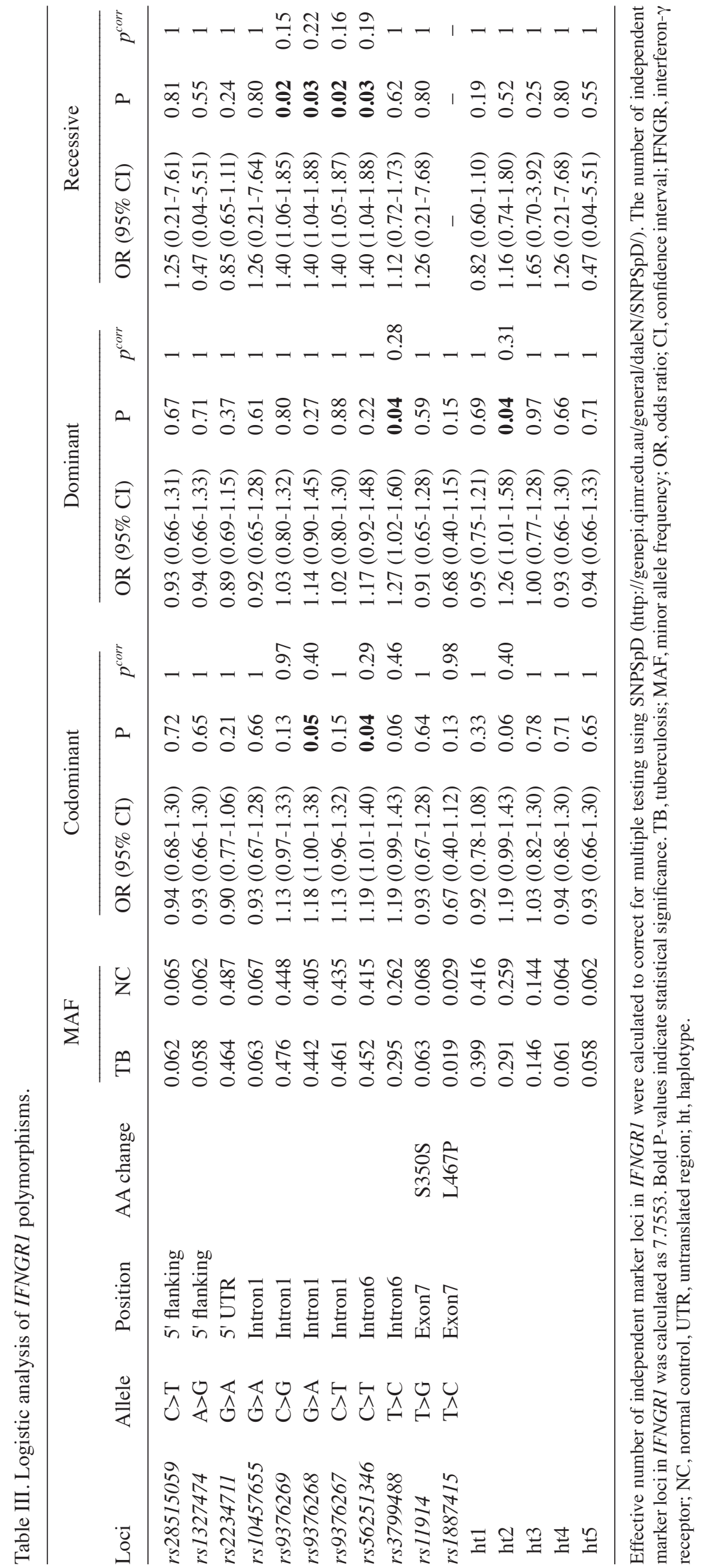




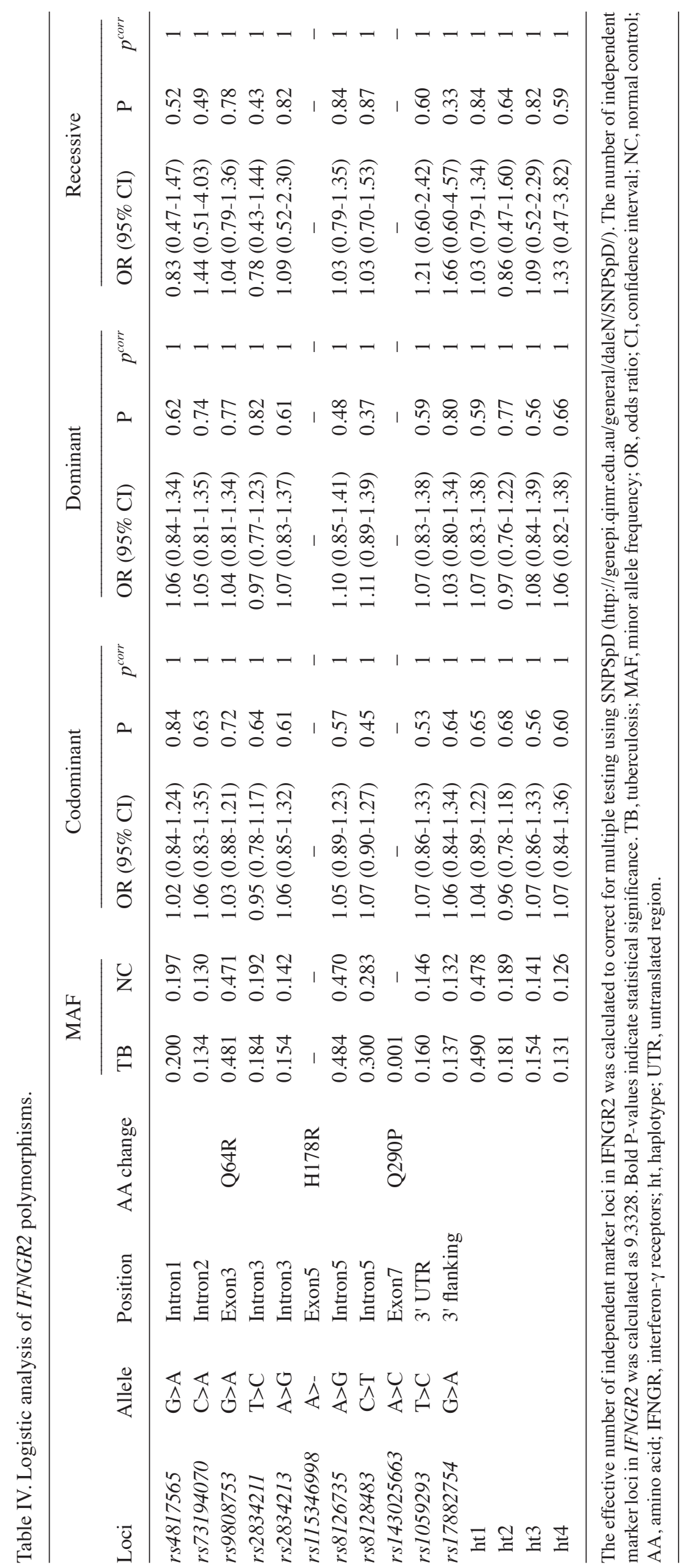


obtained by calculating |D'| and $r^{2}$ values. Among the investigated polymorphisms, ten polymorphisms in IFNGRI and eight in IFGNR2 were used for LD block construction of each gene. The genetic variants $r s 1887415, r s 115346998$ and rs 143025663 were excluded from LD block construction due to its low frequency $(\mathrm{MAF}<5 \%)$. As a result, one LD block was constructed in IFNGRI that contained five major haplotypes (ht), which exhibited a MAF $>5 \%$ (Fig. 1). Among the IFNGRI haplotypes, IFNGRI_ht4 and IFNGRI_ht5 exhibited equivalence with $r s 28515059$ and $r s 1327474$, respectively, and those haplotypes were excluded from the further analysis. In the case of IFNGR2, one LD block was constructed and it contained four major haplotypes, which exhibited a MAF $>5 \%$ (Fig. 2).

Correlation analyses of SNPs in IFNGRI and IFNGR2 with $T B$. The case-control analysis of the correlation between IFNGRI or IFNGR 2 polymorphisms and the risk of TB was conducted (Tables III and IV). The correlation analysis revealed that the two SNPs in IFNGR1, rs9376268 and rs56251346, induced an increased risk for TB under a co-dominant model $(\mathrm{OR}=1.18$ and 1.19; $\mathrm{P}=0.05$ and 0.04 , respectively). The two SNPs exhibited similar genetic effects with a higher level of significance under a recessive model $(\mathrm{OR}=1.40 ; \mathrm{P}=0.03$ for the two SNPs). Along with $r s 9376268$ and $r s 56251346$, two SNPs in intron 1, rs9376269 and rs9376267, also induced an increased risk for TB under a recessive model $(\mathrm{OR}=1.40$; $\mathrm{P}=0.02$ for the two SNPs). However, the level of significance was not retained following the correction for multiple testing in all analysis models $(\mathrm{P}>0.05)$. Polymorphisms in the coding region, rs11914 (S350S) and rs1887415 (L467P), were not associated with an increased risk for TB. In the haplotype analysis, IFNGR1_ht2 exhibited a marginal association with the risk for TB under a dominant model $(\mathrm{P}=0.04)$, although its association was eradicated following the correction for multiple testing. However, no genetic polymorphisms and haplotypes in IFNGR2 exhibited significant correlations with the risk of developing TB.

\section{Discussion}

In previous studies, genetic variations in the genes involved in the IFN- $\gamma$ signaling pathway have been associated with the risk of developing several mycobacterial diseases, particularly TB (13-15). Defects in the proper functioning of IFN- $\gamma$-meditated immune responses is a major cause of disease susceptibility (22). IFN- $\gamma$ activates transcription of a large number of cytokines, including those secreted by macrophages, including IL-12 and TNF- $\alpha$, which have roles in immune responses, thus the appropriate function of the IFNGR appears to be important in host defense against mycobacteria (23).

In the present study, a logistic analysis was conducted to identify a possible significant association between genetic variants in the IFNGR genes and TB in a Korean population. Previous studies have revealed a correlation of the IFNGRI polymorphisms $r s 2234711, r s 1327474$ and $r s 11914$, with TB (Table V) $(13,14,17,24,25)$. Studies in African populations have revealed that the prevalence of TB was lower in African populations with the minor alleles of $r s 11914$ (S350S) and $r s 2234711$, suggesting a protective effect $(\mathrm{OR}=0.66 ; \mathrm{P}=0.022$

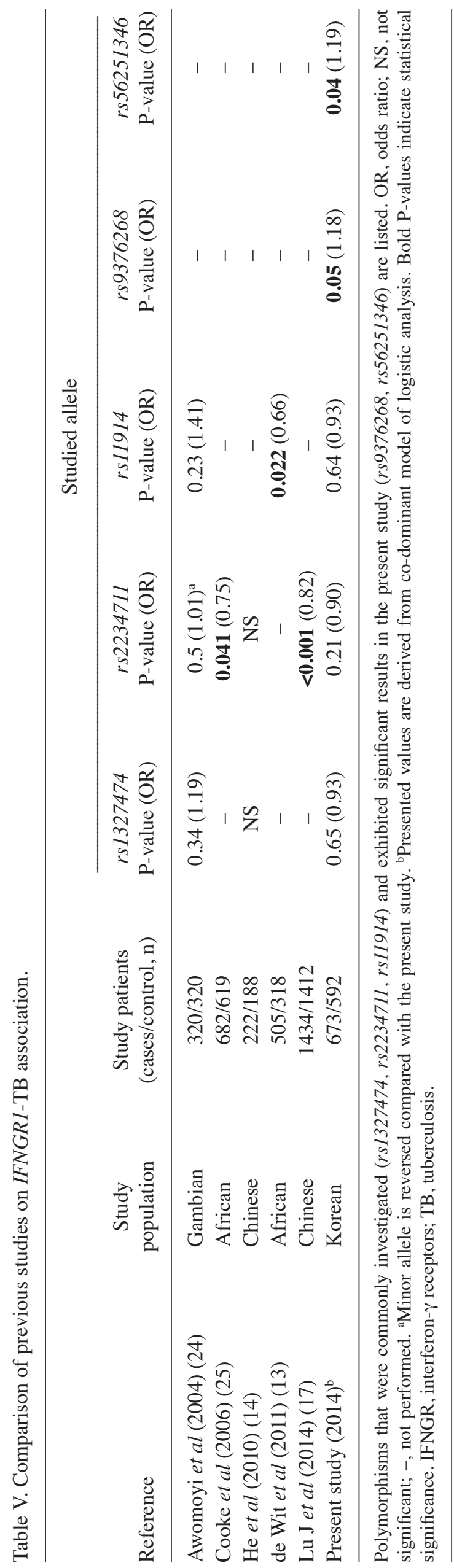




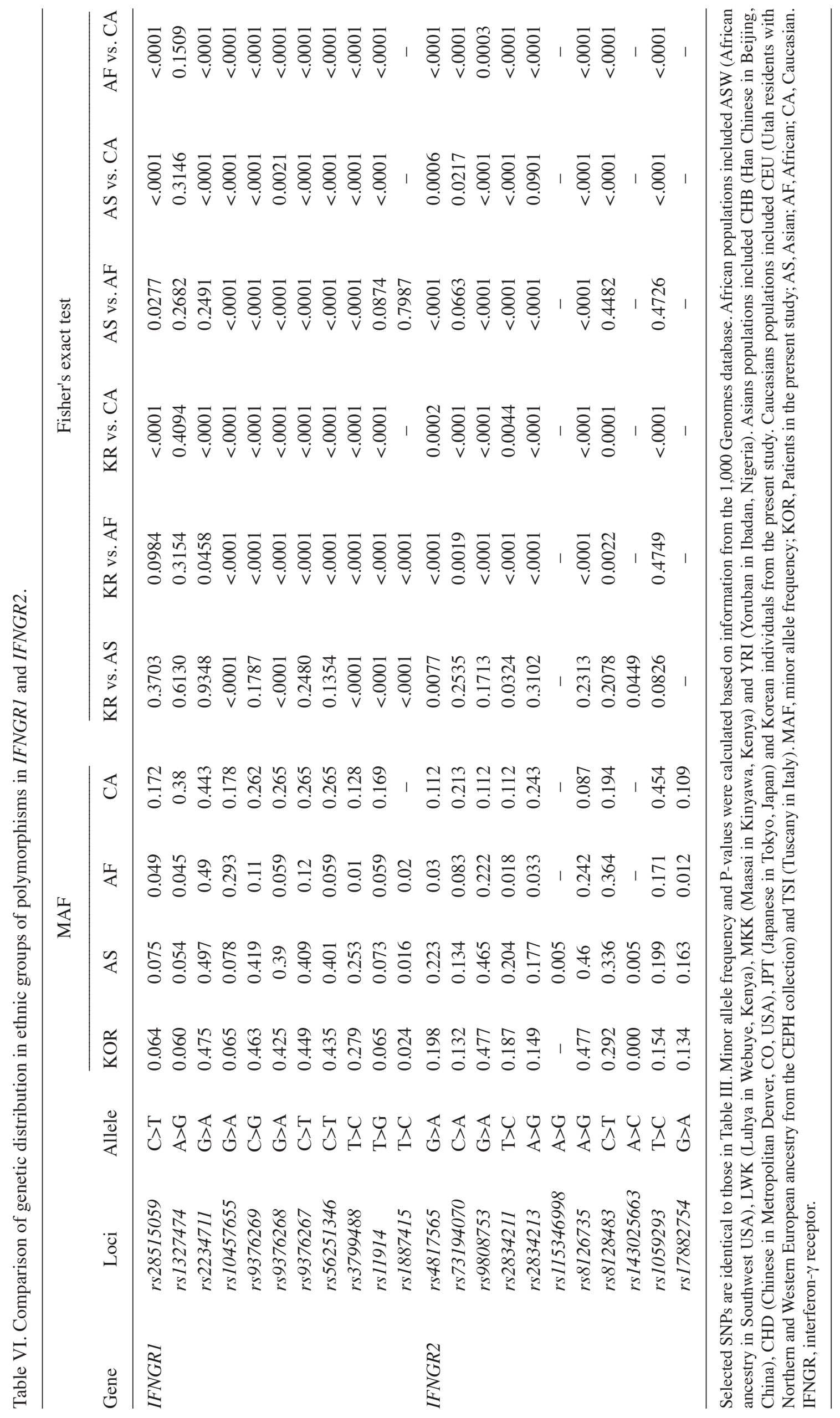


and $\mathrm{OR}=0.75 ; \mathrm{P}=0.041$, respectively $)(13,25)$. The protective effect of $r s 2234711$ on TB prevalence has also been observed in a Chinese population $(\mathrm{OR}=0.82, \mathrm{P}<0.001)(17)$. In another Chinese study, rs 7749300, which revealed a marked LD with $r s 2234711$ and $r s 1327474$, were significantly associated with the risk of $\mathrm{TB}(\mathrm{OR}=3.96 ; \mathrm{P}=0.0003$, from haplotype analysis of three SNPs) (14). However, rs7749300 was not investigated in the present study due to perfect LD with $r s 2234711$ in the 1,000 Genomes database. However, previously demonstrated genetic effects were not replicated in the present study, which may be due to differences in the genetic diversity among the populations. In the case of IFNGR2, two polymorphisms ( $r s 2834213$ and 1059293) exhibited a protective effect against the risk of developing TB (OR=0.69-0.70; $\mathrm{P}=0.0073-0.0088$ ) (26); however, these findings were not replicated in the present study.

In order to investigate whether the present results were due to ethnic differences or not, the genetic composition of IFNGR genes were compared between ethnicities. Frequency analysis and Fisher's exact test were additionally conducted among the four groups, which included a Korean population from the present study, as well as African, Asian and Caucasian populations from the 1,000 Genomes database (Table VI). As a result, the SNP rs11914 exhibited a significant difference in allelic distribution between Korean and African individuals. Genetic compositions of rs11914 in the Japanese and Chinese populations also differed from that of Korean individuals. Along with the rs 11914 SNP, other investigated SNPs, including rs10457655, rs9376269, rs9376268, rs9376267, rs56251346 and $r s 3799488$, have demonstrated a wide degree of frequency variance depending on the populations $(\mathrm{P}<0.05)$.

Previous studies have demonstrated that dysfunction in the IFN- $\gamma$ pathway caused by genetic variation may contribute to a further impairment in cellular immune function in IFN- $\gamma$-mediated diseases, which may increase the susceptibility to disease. A specific promoter polymorphism, rs1327474, and one coding region polymorphism, rs 11914 (S350S), were found to be significantly associated with the risk of arthritis in a European population (27). Other SNPs, rs3799488 and rs10457655, exhibited associations with the risk of rectal cancer prevalence and risk of atopic dermatitis, respectively, in a Caucasian population $(28,29)$.

Of note, functional analysis of IFNGRI identified that the non-synonymous SNP rs1887415 (L467P) does not functionally differ from the wild-type receptors (30). In addition, IFNGR1 L467P has been reported to be associated with the high immunoprotein levels against diseases $(31,32)$. Previous studies of rs1887415 may be a plausible explanation for the protective effect against TB $(\mathrm{OR}=0.63)$ since IFNGRI interacts with the IFN- $\gamma$ immune responses that induce secretion of other cytokines. The association analyses demonstrated that genetic variants in the ligand-binding chain of IFNGR (IFNGR I) affect the IFN- $\gamma$ pathway, although genetic variants in the signal-transducing chain of IFNGR (IFNGR2), including three non-synonymous SNPs (Q64R, H178R, Q290P), do not affect the IFN- $\gamma$ pathway.

In conclusion, a correlation analysis between polymorphisms in IFNGR genes and the risk of TB revealed that four SNPs, rs9376269, rs9376268, rs9376267 and rs56251346, were marginally associated with the development of TB. The present study was the first to report, to the best of our knowledge, the importance of IFNGRI and IFNGR2 as genetic factors in mycobacterial infectious disease, which may prove useful for identifying the etiology of TB in a Korean population.

\section{Acknowledgements}

The present study was supported by the Korean Science and Engineering Foundation funded by the Korean government (grant no. NRF-2011-0021659). The DNA samples were generously provided by Soonchunhyang University, Bucheon Hospital Biobank and a member of the National Biobank of Korea, supported by the Ministry of Health, Welfare and Family Affairs, Republic of Korea.

\section{References}

1. Vannberg FO, Chapman SJ, Khor CC, et al: CD209 genetic polymorphism and tuberculosis disease. PLoS One 3: e1388, 2008.

2. Organization WH: Global tuberculosis report 2012: Geneva, Switzerland: World Moreno S, Jarrin I, Iribarren JA, et al: Incidence and risk factors for tuberculosis in HIV-Guyatt $\mathrm{GH}$, Oxman AD, Vist GE, et al., GRADE: an emerging consensus on rating quality of 0 Guyatt GH, Oxman AD, Kunz R, et al, Incorporating considerations of resources use into 2012

3. Lawn SD and Zumla AI: Tuberculosis. Lancet 378: 57-72, 2011.

4. O'Garra A, Redford PS, McNab FW, Bloom CI, Wilkinson RJ and Berry MP: The immune response in tuberculosis. Annu Rev Immunol 31: 475-527, 2013.

5. Berry MP, Graham CM, McNab FW, et al: An interferon-inducible neutrophil-driven blood transcriptional signature in human tuberculosis. Nature 466: 973-977, 2010.

6. Ferrara G, Losi M, Fabbri LM, Migliori GB, Richeldi L and Casali L: Exploring the immune response against Mycobacterium tuberculosis for a better diagnosis of the infection. Arch Immunol Ther Exp (Warsz) 57: 425-433, 2009.

7. Thye T, Vannberg FO, Wong SH, et al: Genome-wide association analyses identifies a susceptibility locus for tuberculosis on chromosome 18q11.2. Nat Genet 42: 739-741, 2010.

8. Zhang Z, Zhu H, Pu X, et al: Association between tumor necrosis factor alpha-238 G/a polymorphism and tuberculosis susceptibility: a meta-analysis study. BMC Infect Dis 12: 328, 2012.

9. Morris GA, Edwards DR, Hill PC, et al: Interleukin 12B (IL12B) genetic variation and pulmonary tuberculosis: a study of cohorts from The Gambia, Guinea-Bissau, United States and Argentina. PLoS One 6: e16656, 2011.

10. Schroder K, Hertzog PJ, Ravasi $T$ and Hume DA: Interferon-gamma: an overview of signals, mechanisms and functions. J Leukoc Biol 75: 163-189, 2004.

11. Newport MJ, Huxley CM, Huston S, et al: A mutation in the interferon-gamma-receptor gene and susceptibility to mycobacterial infection. N Engl J Med 335: 1941-1949, 1996.

12. Stein CM, Zalwango S, Chiunda AB, et al: Linkage and association analysis of candidate genes for TB and TNFalpha cytokine expression: evidence for association with IFNGR1, IL-10 and TNF receptor 1 genes. Hum Genet 121: 663-673, 2007.

13. de Wit E, van der Merwe L, van Helden PD and Hoal EG: Gene-gene interaction between tuberculosis candidate genes in a South African population. Mamm Genome 22: 100-110, 2011.

14. He J, Wang J, Lei D and Ding S: Analysis of functional SNP in ifng/ifngrl in Chinese Han population with tuberculosis. Scand J Immunol 71: 452-458, 2010.

15. Motsinger-Reif AA, Antas PR, Oki NO, Levy S, Holland SM and Sterling TR: Polymorphisms in IL-1beta, vitamin D receptor Fok1 and Toll-like receptor 2 are associated with extrapulmonary tuberculosis. BMC Med Genet 11: 37, 2010.

16. Fraser DA, Bulat-Kardum L, Knezevic J, et al: Interferon-gamma receptor-1 gene polymorphism in tuberculosis patients from Croatia. Scand J Immunol 57: 480-484, 2003.

17. Lu J, Pan H, Chen Y, et al: Genetic polymorphisms of IFNG and IFNGR1 in association with the risk of pulmonary tuberculosis. Gene 543: 140-144, 2014. 
18. Livak KJ: Allelic discrimination using fluorogenic probes and the 5' nuclease assay. Genet Anal 14: 143-149, 1999.

19. Barrett JC, Fry B, Maller J and Daly MJ: Haploview: analysis and visualization of LD and haplotype maps. Bioinformatics 21: 263-265, 2005

20. Stephens M, Smith NJ and Donnelly P: A new statistical method for haplotype reconstruction from population data. Am J Hum Genet 68: 978-989, 2001.

21. Nyholt DR: A simple correction for multiple testing for single-nucleotide polymorphisms in linkage disequilibrium with each other. Am J Hum Genet 74: 765-769, 2004.

22. Naka I, Patarapotikul J, Hananantachai H, Tokunaga K, Tsuchiya $\mathrm{N}$ and Ohashi J: IFNGR1 polymorphisms in Thai malaria patients. Infect Genet Evol 9: 1406-1409, 2009.

23. Canedo P, Corso G, Pereira F, et al: The interferon gamma receptor 1 (IFNGR1) $-56 \mathrm{C} / \mathrm{T}$ gene polymorphism is associated with increased risk of early gastric carcinoma. Gut 57: 1504-1508, 2008.

24. Awomoyi AA, Nejentsev S, Richardson A, et al: No association between interferon-gamma receptor-1 gene polymorphism and pulmonary tuberculosis in a Gambian population sample. Thorax 59: 291-294, 2004.

25. Cooke GS, Campbell SJ, Sillah J, et al: Polymorphism within the interferon-gamma/receptor complex is associated with pulmonary tuberculosis. Am J Respir Crit Care Med 174: 339-343, 2006
26. Hijikata M, Shojima J, Matsushita I, et al: Association of IFNGR2 gene polymorphisms with pulmonary tuberculosis among the Vietnamese. Hum Genet 131: 675-682, 2012.

27. Nordang GB, Viken MK, Amundsen SS, et al: Interferon regulatory factor 5 gene polymorphism confers risk to several rheumatic diseases and correlates with expression of alternative thymic transcripts. Rheumatology (Oxford) 51: 619-626, 2012.

28. Slattery ML, Lundgreen A, Bondurant KL and Wolff RK: Interferon-signaling pathway: associations with colon and rectal cancer risk and subsequent survival. Carcinogenesis 32: 1660-1667, 2011.

29. Leung DY, Gao PS, Grigoryev DN, et al: Human atopic dermatitis complicated by eczema herpeticum is associated with abnormalities in IFN-gamma response. J Allergy Clin Immunol 127: 965-973, el-e5, 2011.

30. van de Wetering D, de Paus RA, van Dissel JT and van de Vosse E: Functional analysis of naturally occurring amino acid substitutions in human IFN-gammaR1. Mol Immunol 47: 1023-1030, 2010

31. Aoki M, Matsui E, Kaneko H, et al: A novel single-nucleotide substitution, Leu 467 Pro, in the interferon-gamma receptor 1 gene associated with allergic diseases. Int J Mol Med 12: 185-191, 2003.

32. Thye T, Burchard GD, Nilius M, Muller-Myhsok B and Horstmann RD: Genomewide linkage analysis identifies polymorphism in the human interferon-gamma receptor affecting Helicobacter pylori infection. Am J Hum Genet 72: 448-453, 2003. 